\title{
DENSE SUBGROUPS OF LIE GROUPS
}

\author{
DAVID ZERLING
}

\begin{abstract}
Let $G$ be a dense analytic subgroup with compact center of an analytic group $L$. Then there exist closed vector subgroups $W$ and $U$ of $G$ and a $(C A)$ closed normal analytic subgroup $M$ of $G$, which contains the center of $G$, such that $G=M W U, M W \cap U=M \cap W=\{e\}$, and $W U$ is a closed vector subgroup of $G$. Moreover, $L=M W \bar{U}$, where $M W$ is a closed normal analytic subgroup of $L$ and $\bar{U}$ is a toral group, such that $M W \cap \bar{U}$ is finite.
\end{abstract}

1. Introduction. By an analytic group and an analytic subgroup of a Lie group, we mean a connected Lie group and a connected Lie subgroup, respectively. If $G$ and $H$ are Lie groups and $\varphi$ is a one-to-one (continuous) homomorphism from $G$ into $H, \varphi$ will be called an immersion. $\varphi$ will be called closed or dense, as $\varphi(G)$ is closed or dense in $H . G_{0}$ and $Z(G)$ will denote the identity component group and center of $G$, respectively.

If $G$ is an analytic group, $A(G)$ will denote the Lie group of all (bicontinuous) automorphisms of $G$, topologized with the generalized compact-open topology. $G$ will be called $(C A)$ if $I(G)$, the Lie group of all inner automorphisms of $G$, is closed in $A(G)$. It is well known that $G$ is $(C A)$ if and only if its universal covering group is $(C A)$.

If $G$ is a normal analytic subgroup of an analytic group $H$, then each element $h$ of $H$ induces an automorphism of $G$, namely, $g \mapsto h g h^{-1}$. We will denote this homomorphism from $H$ into $A(G)$ by $\rho_{G H} . I_{H}(h)$ will denote the inner automorphism of $H$ determined by $h \in H$. More generally, if $A$ is a subset of $H, I_{H}(A)$ will denote the set of all inner automorphisms of $H$ determined by elements of $A . I_{H}(H)$ will be written as $I(H)$, and the mapping $h \mapsto I_{H}(h)$ of $H$ onto $I(H)$ will be denoted by $I_{H}$.

If $N$ is an analytic group and $H$ is an analytic subgroup of $A(N)$, then $N$ (5) $H$ will denote the semidirect product of $N$ and $H$. On the other hand, if $G$ is an analytic group containing a closed normal analytic subgroup $N$ and a closed analytic subgroup $H$, such that $G=N H, N \cap H=\{e\}$, and such that the restriction of $\rho_{N G}$ to $H$ is one-to-one, we will frequently identify $G$ with $N$ (s) $\rho_{N G}(H)$ and $H$ with $\rho_{N G}(H)$, that is, we may write $G=N$ (5) $H$.

In Zerling [3] we proved the following theorem.

MAIN STRUCTURE THEOREM. Let $G$ be a non- $(C A)$ analytic group. Then there exist a $(C A)$ analytic group $M$, a toral group $T$ in $A(M)$, and a dense vector

Presented to the Society, January 27, 1977; received by the editors July 27, 1976.

AMS (MOS) subject classifications (1970). Primary 22E15; Secondary 57E05.

(c) American Mathematical Society 1977 
subgroup $V$ of $T$, such that:

(i) $H=M$ (5) $T$ is a (CA) analytic group.

(ii) $G$ is isomorphic to the dense analytic subgroup $M$ (5) $V$ of $H$.

(iii) $Z(G)$ is contained in $M$.

(iv) $Z_{0}(G)=Z_{0}(H)$, and $\pi(Z(H))$ is finite, where $\pi$ is the natural projection of $H$ onto $T$. Moreover, if $G / Z(G)$ is homeomorphic to Euclidean space, then $Z(G)=Z(H)$.

(v) Each automorphism $\sigma$ of $G$ can be extended to an automorphism $\varepsilon(\sigma)$ of $H$, such that $\varepsilon: A(G) \rightarrow A(H)$ is a closed immersion.

We will now use this theorem in $\$ 2$ to obtain our main results.

\section{Main results.}

LEMMA. Let us maintain the notation of the main structure theorem and let $f$ : $G \rightarrow L$ be a dense immersion of $G$ into an analytic group $L$. If $Z(G)$ is compact, then $f(M)$ is closed in $L$.

Proof. Since $G$ is non- $(C A)$ we can appeal to Goto [1]: Let $N$ be a maximal analytic subgroup of $I(G)$, which contains the commutator subgroup of $I(G)$ and is closed in $A(G)$. Then there is a closed vector subgroup $V^{\prime}$ of $I(G)$, such that $I(G)=N V^{\prime}, N \cap V^{\prime}=\{e\}$, and $\overline{I(G)}=N \cdot \bar{V}^{\prime}$, where $T^{\prime}=\bar{V}^{\prime}$ is a toral group. Moreover, $N \cap T^{\prime}$ is finite, and the space of $\overline{I(G)}$ is diffeomorphic to the product space $N \times T^{\prime}$.

In the proof of the main structure theorem in Zerling [3], $H$ is constructed in such a way that $\rho_{G H}(M)=N, \rho_{G H}(V)=V^{\prime}$, and $\rho_{G H}(T)=T^{\prime}$. Also, $\rho_{G H}$ is $1-1$ on $T$.

Since $Z(G)$ is of finite index in $Z(H)$ from Zerling [4, Lemma 2.1], $Z(H)$ is also compact. Consider the normal analytic subgroup $\overline{f(M)} \cdot f(V)$ of $L$. Since the inner automorphic action of $f(V)$ on $\overline{f(M)}$ is effective, we have the Lie group $P=\overline{f(M)}$ (5) $V$. The image of each one-parameter subgroup of $V$ under $\rho_{G P}$ is not closed in $A(G)$. Therefore, since $G$ is dense in $P$, we see from Lemma 3.1 of Zerling [4] that the closure of $V$ in $A(\overline{f(M)})$ is a toral group, which we will denote by $T_{1}$.

Now let $Q=\overline{f(M)}$ (s) $T_{1}$. Then $\rho_{G Q}\left(T_{1}\right)=T^{\prime}$, and since $\tau_{1} \cdot(m, v) \cdot \tau_{1}^{-1}=$ $\left(\tau_{1}(m), v\right)$ for all $(m, v)$ in $G$, we see that $\rho_{G Q}$ is $1-1$ on $T_{1}$. Since $\rho_{G H}(T)=T^{\prime}$, and $\rho_{G H}$ is 1-1 on $T$, we have that $\rho_{G Q}^{-1} \circ \rho_{G H}$ is an isomorphism of $T$ onto $T_{1}$. Hence, $H=M$ (s) $T$ is a dense $(C A)$ analytic subgroup of $Q$. Since $Z(H)$ is compact, we may appeal to van Est [2, Theorem 2.2.1] to conclude that $H \cong Q$. Hence, $f(M)=\overline{f(M)}$ and our theorem is proved.

THEOREM. Let $f: G \rightarrow L$ be a proper dense inmersion of an analytic group $G$ into an analytic group L. Suppose $Z(G)$ is compact. Then there exist closed vector subgroups $W$ and $U$ of $G$ and $a(C A)$ closed normal analytic subgroup $M$ of $G$, which contains $Z(G)$, such that:

(i) $G=M W U, M W \cap U=M \cap W=\{e\}$, and $W U$ is a closed vector subgroup of $G$. 
(ii) $L=f(M W) \cdot \overline{f(U)}$, where $f(M W)$ is a closed normal analytic subgroup of $L$ and $\overline{f(U)}$ is a toral group. Moreover, $f(M W) \cap \overline{f(U)}$ is finite, and $f(W) \cap(f(M) \cdot \overline{f(U)})=f(W) \cap Z(L)=\{e\}$.

(iii) If $L$ is $(C A)$ and $Z(L)$ is compact, then $W=\{e\}$.

Proof. Since $Z(G)$ is compact, we can conclude from van Est [2] that $G$ is non- $(C A)$. We will now maintain the notation of the main structure theorem, as well as the notation in the proof of the above lemma.

Since $Z(G)$ is compact, we know from the above lemma that there exists a maximal analytic subgroup $J$ of $G$ which contains $M$, such that $f(J)$ is closed in $L$. Then from Goto [1] there exists a closed vector subgroup $U$ of $G$ such that $G=J U, J \cap U=\{e\}$. Moreover, $L=f(J) \cdot \overline{f(U)}$, where $\overline{f(U)}$ is a toral group and $f(J) \cap \overline{f(U)}$ is finite.

In the proof of Goto's theorem [1], applied to $I(G), T^{\prime}$ is a closed central subgroup of an arbitrarily fixed maximal compact subgroup $K$ of $\overline{I(G)}$. We will assume that $K$ has been selected so that it contains $\rho_{G L}(\overline{f(U)})$.

Now let $\pi: G \rightarrow V$ be the natural projection and let $W=\pi(J)$. Then $W$ is a closed vector subgroup of $V$ and since $J$ contains $M$ we see that $J=M \cdot W$, $M \cap W=\{e\}$. Therefore,

$$
L=f(J) \cdot \overline{f(U)}=f(M) \cdot f(W) \cdot \overline{f(U)}=f(M) \cdot \overline{f(U)} \cdot f(W),
$$

where $f(M) \cdot \overline{f(U)}$ is a closed normal analytic subgroup of $L$. Since $\overline{f(U)} \cap$ $f(J)$ is finite and contained in $f(G)$, it is contained in $f(M)$. Hence, if $(f(M) \cdot \overline{f(U)}) \cap f(W) \neq\{e\}$, then $f(w)=f(m) \cdot x, x \in \overline{f(U)}$, and so $x=$ $f(m)^{-1} \cdot f(w)$. Hence, $x \in \overline{f(U)} \cap f(J)$, which is contained in $f(M)$. By the uniqueness of the decomposition in $J$, we have $w=e$. So $L=(f(M) \cdot \overline{f(U)})$ - $f(W),(f(M) \cdot \overline{f(U)}) \cap f(W)=\{e\}$. Moreover, $f(W) \cap Z(L)=\{e\}$, since $f(J) \cap Z(L)$ is contained in $f(M)$, and $W \cap M=\{e\}$. Hence, $L=$ $(f(M) \cdot \overline{f(U)})$ (s) $f(W)$.

We now show that $W U$ is a closed vector subgroup of $G=M W U$. Let $\varphi$ : $W \rightarrow A(M U)$ be given by $\varphi(w)(m u)=w(m u) w^{-1}$. Since $W \cap Z(G)=\{e\}$, $\varphi$ is an immersion and so $G=M U$ (5) $W$. Since the image of each one-parameter subgroup of $W$ under $I_{G}$ is not closed in $A(G)$, we see from Zerling [4] that $\overline{\varphi(W)}$ is a toral group.

Let $u \in U$. Then $I_{G}(\varphi(w) \cdot u)=I_{G}\left(w u w^{-1}\right)=I_{G}(u)$, since $I_{G}(w) \in T^{\prime}$ commutes with $I_{G}(u) \in \rho_{G L}(\overline{f(U)})$ because of our selection of $K$ above. Hence $\sigma(u) \cdot u^{-1} \in Z(G)$ for all $\sigma \in \overline{\varphi(W)}$. Since $Z(G)$ is a closed central subgroup of $M U$ and each element of $\overline{\varphi(W)}$ keeps $Z(G)$ elementwise fixed, we see from Zerling [3, Lemma 2.2] that $\sigma(u)=u$ for all $\sigma \in \overline{\varphi(W)}$. Therefore, $U W$ is a closed vector subgroup of $G$.

Now suppose that $L$ is $(C A)$ and $Z(L)$ is compact. As we did above, we can show that the closure of $f(W)$ in $A(f(M) \cdot \overline{f(U)})$ is a toral group, call it $T_{2}$. Then $L$ is properly dense in $(f(M) \cdot \overline{f(U)})$ (5) $T_{2}$. This is a contradiction from van Est [2]. Hence $W=\{e\}$. This completes the proof our theorem. 
3. An example. The following example shows that in the above theorem $W$ need not be $\{e\}$, even if $L$ is $(C A)$. Let $G=M V$ be any non- $(C A)$ analytic group and suppose that the dimension of $V$ is at least two. Such a group $G$ can easily be obtained by a slight modification of the example in Zerling [3].

We continue with our previous notation and let $S=G$ (s) $T^{\prime}$. Since $V$ is dense in $T$ we can select an element $v_{0} \in V$ such that $v_{0}$ generates a dense subgroup of $T$. Then $v_{0}^{\prime}=I_{G}\left(v_{0}\right)$ generates a dense subgroup of $T^{\prime}$. Let $D$ denote the subgroup of $S$ generated by $\left(v_{0}, v_{0}^{\prime-1}\right)$. Then $D$ is a free discrete central subgroup of $S$ and $L=S / D$ is a $(C A)$ analytic group for which $g \mapsto(g, e) D$ is a dense immersion $f$ of $G$ into $L$; see Zerling [4, the proof of Theorem 2.2].

Next let $V_{\lambda}$ be the one-dimensional vector subgroup of $V$ containing $v_{0}$, and let $V_{\mu}$ be a vector subgroup of $V$ such that $V=V_{\lambda} \cdot V_{\mu}, V_{\lambda} \cap V_{\mu}=\{e\}$. Let $\delta: S \rightarrow L$ be the canonical projection. Then $G=M V_{\mu} V_{\lambda}, f\left(M V_{\mu}\right)$ is closed in $L$ and $\overline{f\left(V_{\lambda}\right)}=f\left(V_{\lambda}\right) \cdot \delta\left(T^{\prime}\right)$ is a toral group. $L=f\left(M V_{\mu}\right)$ . $\overline{f\left(V_{\lambda}\right)}$, and $f\left(M V_{\mu}\right) \cap \overline{f\left(V_{\lambda}\right)}$ is trivial. Hence $V_{\mu}=W \neq\{e\}$.

\section{BIBLIOGRAPHY}

1. M. Goto, Analytic subgroups of GL(n, R), Tôhoku Math. J. (2) 25 (1973), 197-199. MR 48 \#463.

2. W. T. van Est, Dense imbeddings of Lie groups, Nederl. Akad. Wetensch. Proc. Ser. A 54 = Indag. Math. 13 (1951), 321-328. MR 13, 432.

3. D. Zerling, Some theorems on (CA) analytic groups, Trans. Amer. Math. Soc. 205 (1975), 181-192. MR 51 \# 802.

4. __ Some theorems on (CA) analytic groups. II, Tôhoku Math. J. (to appear).

Department of Mathematics and Physics, Philadelphia College of Textiles and Science, Philadelphia, Pennsylvania 19144. 